Provided for non-commercial research and education use. Not for reproduction, distribution or commercial use.

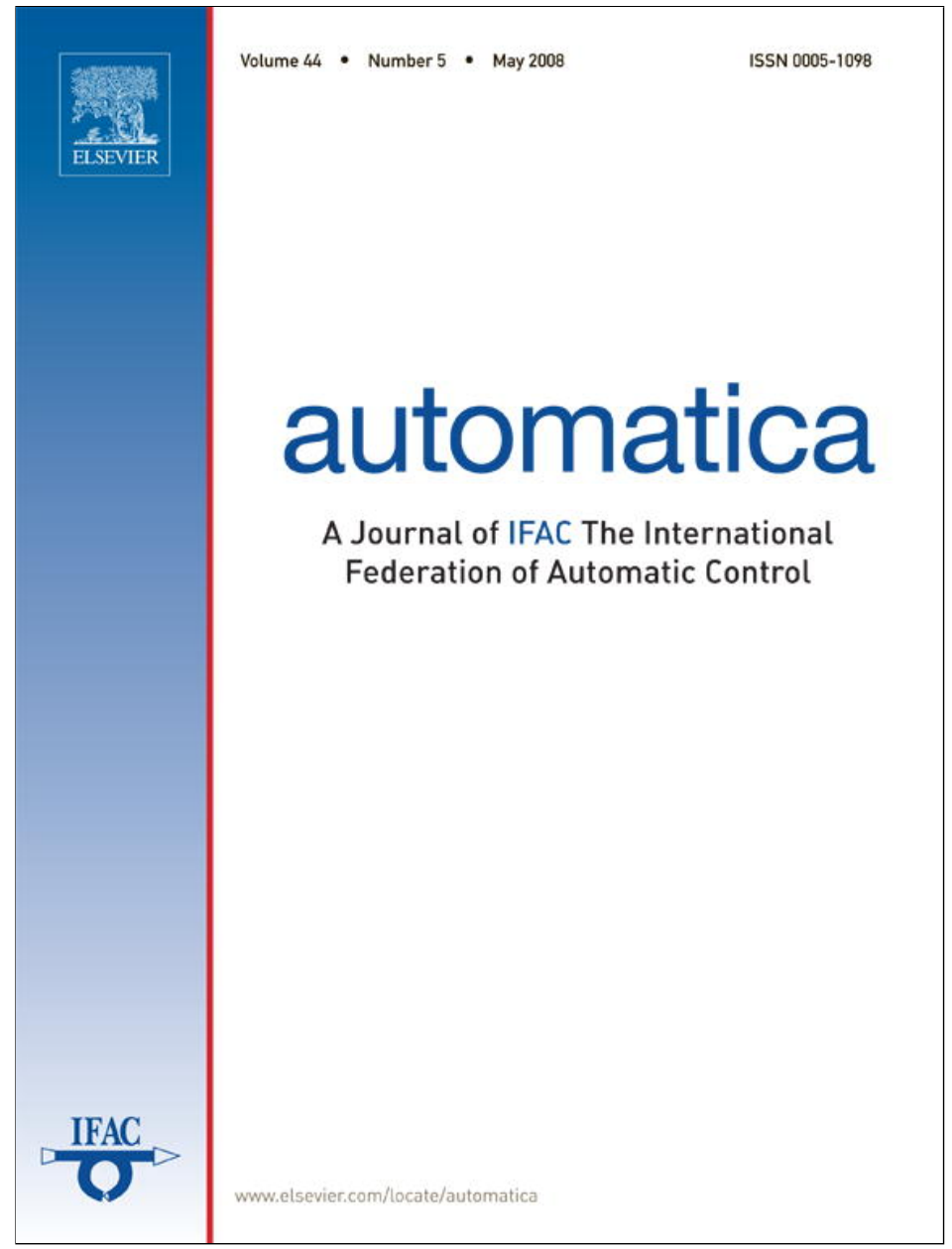

This article appeared in a journal published by Elsevier. The attached copy is furnished to the author for internal non-commercial research and education use, including for instruction at the authors institution and sharing with colleagues.

Other uses, including reproduction and distribution, or selling or licensing copies, or posting to personal, institutional or third party websites are prohibited.

In most cases authors are permitted to post their version of the article (e.g. in Word or Tex form) to their personal website or institutional repository. Authors requiring further information regarding Elsevier's archiving and manuscript policies are encouraged to visit:

http://www.elsevier.com/copyright 


\title{
On stability, $L_{2}$-gain and $H_{\infty}$ control for switched systems ${ }^{\text {th }}$
}

\author{
Jun Zhao ${ }^{\mathrm{a}, \mathrm{b}, *}$, David J. Hill ${ }^{\mathrm{b}}$ \\ ${ }^{a}$ Key Laboratory of Integrated Automation of Process Industry, Ministry of Education of China, Northeastern University, Shenyang, 110004, PR China \\ ${ }^{\mathrm{b}}$ Department of Information Engineering, Research School of Information Sciences and Engineering, The Australian National University, \\ Canberra ACT 0200, Australia
}

Received 3 January 2007; received in revised form 30 May 2007; accepted 7 October 2007

Available online 5 March 2008

\begin{abstract}
This paper addresses the issues of stability, $L_{2}$-gain analysis and $H_{\infty}$ control for switched systems via multiple Lyapunov function methods. A concept of general Lyapunov-like functions is presented. A necessary and sufficient condition for stability of switched systems is given in terms of multiple generalized Lyapunov-like functions, which enables derivation of improved stability tests, an $L_{2}$-gain characterization and a design method for stabilizing switching laws. A solution to the $H_{\infty}$ control problem for switched systems is also provided.
\end{abstract}

(c) 2008 Elsevier Ltd. All rights reserved.

Keywords: Switched systems; Stability; Multiple Lyapunov functions; $L_{2}$-gain; $H_{\infty}$ control

\section{Introduction}

Switched systems constitute a special class of hybrid systems which contain both continuous dynamics and discrete dynamics. Switched systems arise in engineering practice where several dynamical system models are required to model an engineering system due to the various jumping parameters and changing environmental factors. The widespread applications of switched systems are also motivated by increasing performance requirements in control, especially in the presence of large uncertainties or disturbances. A good example is the so-called intelligent control design where switching between different controllers is a basic mechanism. It is now well understood that switched controller systems, as a special type of switched systems, can provide a control solution that guarantees stability and good performance when no single controller is effective-see for instance, Narendra, Driollet, Feiler, and George (2003), Savkin, Skafidas, and Evans (1999),

\footnotetext{
This paper was not presented at any IFAC meeting. This paper was recommended for publication in revised form by Associate Editor Maria Elena Valcher under the direction of Editor Roberto Tempo.

* Corresponding author at: Department of Information Engineering, Research School of Information Sciences and Engineering, The Australian National University, Canberra ACT 0200, Australia. Tel.: +61 2 61258669; fax: +61261258660.

E-mail addresses: jun.zhao@anu.edu.au (J. Zhao), David.Hill@anu.edu.au (D.J. Hill).
}

and Skafidas, Evans, Savkin, and Petersen (1999). As a specific situation, many complex nonlinear continuous or discrete systems that are not stabilizable by a single continuous or discrete controller can be stabilized by switching between finitely many controllers (Cronin \& Spong, 2003; Ishii \& Francis, 2002; Kosmatopoulos \& Ioannou, 2002; Zhao \& Spong, 2001).

The main concern in the study of switched systems is the issue of stability (Bacciotti, 2004; Cheng, Guo, Lin, \& Wang, 2005; Haddad \& Chellaboina, 2001; Ji, Wang, \& Guo, 2007; Margaliot, 2006; Sun \& Ge, 2005; Zhao \& Dimirovski, 2004). This issue is very difficult to deal with due to the hybrid nature of switched systems operation. The existence of a common Lyapunov function for all subsystems was shown to be a necessary and sufficient condition for a switched system to be asymptotically stable under an arbitrary switching law (see Liberzon (2003) and references therein). A considerable number of techniques have been proposed to construct such a Lyapunov function (see Decarlo, Branicky, Pettersson, and Lennartson (2000), Liberzon and Morse (1999), and Ooba and Funahashi (1997) for instance). Most switched systems in practice, however, do not possess a common Lyapunov function, yet they still may be asymptotically stable under some properly chosen switching law. A typical switching law is given by the convex combination method (Liberzon \& Morse, 1999). In many cases, for a switching law either pre-given or to be 
designed, each subsystem has its own Lyapunov-like function which behaves as usual Lyapunov function only when this subsystem is active. A common Lyapunov-like function for all subsystems, which obviously qualifies as a usual Lyapunov function, may not exist or be difficult to find. Therefore, the multiple Lyapunov function technique proposed by Peleties and DeCarlo (1991) and later generalized by Branicky (1998) and Hou, Michel, and Ye (1996), and Ye, Michel, and Hou (1998) has proven to be a powerful and effective tool for finding such a switching law. Many results in this direction have been available (for example, see EI-Farra, Mhaskar, and Christofides (2005) and Hespanha, Liberzon, Angeli, and Sontag (2005)).

The key point of these conditions of multiple Lyapunov function methods is the nonincreasing requirement on any Lyapunov function over the "switched on" time sequence of the corresponding subsystem. However, this is usually hard to check in its full generality. Thus, in order to apply the multiple Lyapunov function methods, connecting adjacent Lyapunov functions at switching points is a commonly accepted strategy. This can be typically achieved by choosing the switching law according to the "min-switching" strategy of all Lyapunov functions (Liberzon \& Morse, 1999), which is a special case of Branicky's nonincreasing condition, but easy to design and realize. As a result, a globally piecewise defined continuous Lyapunov function is formed. Switched Lyapunov function method (Daafouz, Riedinger, \& Iung, 2002) is another special case of Branicky's multiple Lyapunov function technique.

Turning to the $L_{2}$-gain analysis and $H_{\infty}$ control problems, these subjects have been extensively explored in terms of algebraic Riccati inequalities for linear systems and Hamilton-Jacobi inequalities for nonlinear systems. These results, together with many other extensions of the basic results (Hill \& Moylan, 1976, 1977), are summarized in the recent books (Helton \& James, 1999; van der Schaft, 2000). However, the $L_{2}$-gain analysis and $H_{\infty}$ control problems have been rarely addressed for switched systems. As an open problem, the $L_{2}$-gain that all linear subsystems share was put forward in Hespanha and Morse (1999). The $L_{2}$-induced norm of switched linear systems composed of Hurwitz stable subsystems was considered and a weighted gain was reached under a large dwell time condition (Hespanha, 1998). Using the dwell time concept, another result of disturbance attenuation for both switched linear and nonlinear systems was reported by Zhai, Ho, Yasuda, and Michel (2001). Stability and $L_{2-}$ gain analysis were addressed for switched delay systems by the average dwell time method (Sun, Zhao, \& Hill, 2006). In these results, multiple Lyapunov functions are used but they are not necessarily connected to each other at switching points. In other words, "jumps" of adjacent Lyapunov functions at switching times may occur, but this results in a weaker attenuation property - a weighted disturbance attenuation level (Zhai et al., 2001). In order to have a standard form of attenuation level, which has been commonly accepted in the control area, multiple Lyapunov functions based on the "min-switching" strategy of all Lyapunov functions have been applied (Liberzon, 2003; Liberzon \& Morse, 1999). As a result, the "jumps" are completely eliminated. Of course, this elimination of "jumps" is only possible when some strong assumptions are imposed. There seems a gap between maintaining a standard attenuation level and the use of multiple Lyapunov functions that are not necessarily connected at switching times.

Some other results concerning $L_{2}$-gains and $H_{\infty}$ control for certain classes of hybrid systems or hybrid control are also available. $H_{\infty}$ control for uncertain discrete switched systems was addressed in Lin and Antsaklis (2003), Xie, Wang, Hao, and Xie (2004), and Zhang, Shi, Boukas, and Wang (2006). An approximated solution to $H_{\infty}$ control for a class of nonlinear processes using multiple linear models was proposed in Banerjee, Arkun, Pearson, and Ogunnaike (1997). $H_{\infty}$ analysis and control for discrete piecewise linear systems were explored when the switching region is prescribed (Cuzzola \& Morari, 2002). BIBO stability was addressed for discrete switched linear systems in Michaletzky and Gerencser (2002). Again, connection of adjacent Lyapunov functions or just use of a single Lyapunov function is a fundamental requirement in these results.

This paper studies the problems of stability, $L_{2}$-gain analysis and $H_{\infty}$ control for switched systems via multiple Lyapunov function methods. The paper is organized as follows. Section 2 gives the preliminaries. In Section 3 we present a notion of generalized Lyapunov-like functions and derive a necessary and sufficient condition for stability in terms of multiple generalized Lyapunov-like functions. An algebraic condition and design method for stabilizing switching laws are also given. Section 4 is devoted to $L_{2}$-gain analysis and design. The $H_{\infty}$ control problem is explored in Section 5. Finally, Section 6 contains the concluding remarks. Earlier version of part of the results was presented in Zhao and Hill (2005).

\section{Preliminaries}

We consider the class of switched nonlinear systems of the form:

$\dot{x}=f_{\sigma}\left(x, u_{\sigma}\right)$,

$y=h_{\sigma}(x)$,

where $x \in R^{n}$ is the state, $\sigma: R_{+}=[0, \infty) \rightarrow M=$ $\{1,2, \ldots, m\}$ is the switching signal which is assumed to be a piecewise continuous (from the right) function depending on time or state or both. $m$ is the number of models (called subsystems) of the switched system. $u_{i}$ and $h_{i}(x), i \in M$, are the input vector and output vector of the $i$ th subsystem respectively. $f_{i}(x, u)$ and $h_{i}(x)$ are assumed to be continuous with $f_{i}(0,0)=0$ and $h_{i}(0)=0, i \in M$. Here, we adopt the standard notations from Branicky (1998) and Hou et al. (1996). The switching signal $\sigma$ can be characterized by a switching sequence (with abuse use of terms, also called a switching law):

$\Sigma=\left\{x_{0} ;\left(i_{0}, t_{0}\right),\left(i_{1}, t_{1}\right), \ldots,\left(i_{n}, t_{n}\right), \ldots \mid i_{n} \in M, n \in N\right\}$,

in which $t_{0}$ is the initial time, $x_{0}$ is the initial state and $N$ is the set of nonnegative integers. When $t \in\left[t_{k}, t_{k+1}\right), \sigma(t)=i_{k}$, that is, the $i_{k}$ th subsystem is active. Therefore, the trajectory $x(t)$ of the switched system (1) is the trajectory of the $i_{k}$ th subsystem when $t \in\left[t_{k}, t_{k+1}\right)$. The solution $x(t)$ of the system 
(1) is assumed to exist and to be unique. Let $x_{k}$ denote $x\left(t_{k}\right)$. The switching sequence $\Sigma$ may or may not be infinite. In the finite case, we may take $t_{n+1}=\infty$, with all further definitions and results still valid.

We assume that the state of the system (1) does not jump at the switching instants, i.e., the trajectory $x(t)$ is everywhere continuous. Also, we assume $i_{k} \neq i_{k+1}$ for any $k$.

For any $j \in M$, let

$\Sigma \mid j=$

$\left\{t_{j_{1}}, t_{j_{1}+1}, t_{j_{2}}, t_{j_{2}+1}, \ldots, t_{j_{n}}, t_{j_{n}+1}, \ldots: i_{j_{q}}=j, q \in N\right\}$

be the sequence of switching times when the $j$ th subsystem is switched on or switched off. For a given strictly increasing sequence of times $T=\left\{t_{0}, t_{1}, \ldots, t_{n}, \ldots,\right\}$, the interval completion $I(T)$ is the set $I(T)=\bigcup_{j \in N}\left[t_{2 j}, t_{2 j+1}\right)$. Let $E(T)=\left\{t_{0}, t_{2}, t_{4}, \ldots,\right\}$ denote the even sequence of $T$. Therefore,

$E(\Sigma \mid j)=\left\{t_{j_{1}}, t_{j_{2}}, \ldots, t_{j_{n}}, \ldots, n \in N\right\}$

is the "switched on" times of the $j$ th subsystem.

Assumption 2.1. $\sigma$ has finite number of switchings on any finite interval of time.

This is a standard assumption in the literature to rule out Zeno behavior for all types of switching (Liberzon, 2003). How to identify or avoid Zeno phenomena is a challenging topic which is beyond the scope of this paper. Some effective methods can be found, for example, in Ceragioli (2006).

Unless otherwise stated, all results hold for the switching law $\Sigma$ given by (2).

Notations: $\|\cdot\|$ : the Euclidean norm; $\arg \min S:$ the index of minimum element of the ordered set $S$; $\bigcup$ the union operator of sets; class $\mathcal{K}$ function: a continuous function defined on $[0, \infty)$, which is increasing and vanishes at the origin; $C^{1}\left[R^{n}, R_{+}\right]$: the space of continuously differentiable functions from $R^{n}$ to $R_{+} ; C\left[R_{+}, R_{+}\right]$: the space of continuous functions from $R_{+}$to $R_{+}$.

\section{Stability}

In this section, we study stability of the system (1). We derive a necessary and sufficient condition for stability by extending the existing multiple Lyapunov function methods.

\subsection{Overview of multiple Lyapunov function methods}

First of all, we give a brief review of multiple Lyapunov function methods.

Definition 3.1 (Lyapunov-like Function (Branicky, 1998; Peleties \& DeCarlo, 1991)). Given a strictly increasing sequence of times $T$, a function $V \in C^{1}\left[R^{n}, R_{+}\right]$with $V(0)=0$ is called a Lyapunov-like function for the vector field $f$ and the associated trajectory $x(t)$ over $T$ if

(i) $\dot{V}(x(t)) \leq 0$ for all $t \in I(T)$, (ii) $V$ is nonincreasing on $E(T)$.
Theorem 3.2 (Peleties \& DeCarlo, 1991). Consider the system (1) with $u_{i} \equiv 0$. Assume that for each $j, V_{j}$ is a Lyapunovlike function for the $j$ th subsystem vector field $f_{j}$ and the associated trajectory over $T=\Sigma \mid j$. If there exists a constant $\rho>0$ with the property that for any two switching times $t_{p}, t_{q} \in E(\Sigma \mid j), p<q$, we have

$V_{\sigma\left(t_{q}\right)}\left(x\left(t_{q+1}\right)\right)-V_{\sigma\left(t_{p}\right)}\left(x\left(t_{p+1}\right)\right) \leq-\rho\left\|x\left(t_{p+1}\right)\right\|^{2}$,

then the origin of the system (1) is globally asymptotically stable.

Theorem 3.3 (Branicky, 1998). Consider the system (1) with $u_{i} \equiv 0$. If for each $j, V_{j}$ is a Lyapunov-like function for the $j$ th subsystem vector field $f_{j}$ and the associated trajectory over $T=\Sigma \mid j$, then the origin of the system (1) is stable.

The stability result has been generalized to hold for weak Lyapunov-like functions (Ye et al., 1998):

Definition 3.4 (Weak Lyapunov-like Function (Hou et al., 1996; Ye et al., 1998)). A function $V \in C^{1}\left[R^{n}, R_{+}\right]$with $V(0)=0$ is called a weak Lyapunov-like function for the vector field $f$ and the associated trajectory $x(t)$ over a strictly increasing sequence of times $T$ if

(i) there exists a function $\phi \in C\left[R_{+}, R_{+}\right]$satisfying $\phi(0)=$ 0 , such that $V(x(t)) \leq \phi\left(V\left(x\left(t_{2 j}\right)\right)\right)$ for all $t \in\left(t_{2 j}, t_{2 j+1}\right)$ and all $j \in N$, (ii) $V$ is nonincreasing on $E(T)$.

Theorem 3.5 (Hou et al., 1996; Ye et al., 1998). Consider the system (1) with $u_{i} \equiv 0$. Iffor each $j, V_{j}$ is a radially unbounded weak Lyapunov-like function for the $j$ th subsystem vector field $f_{j}$ and the associated trajectory over $T=\Sigma \mid j$, then the origin of the system (1) is stable.

In all the above-mentioned results using multiple Lyapunov functions, a fundamental common assumption is the nonincreasing condition of $V$ on $E(T)$. This is obviously quite conservative and hard to check. We will relax this restriction in the next subsection to allow some increase of $V$ on $E(T)$.

\subsection{Necessary and sufficient condition}

In order to relax the nonincreasing condition of $V$-functions on $T=\Sigma \mid j$, we first generalize the concept of weak Lyapunov-like functions.

Definition 3.6. A continuous function $V: R^{n} \rightarrow[0, \infty)$ is called a generalized Lyapunov-like function if the condition (i) in Definition 3.4 holds.

Obviously, weak Lyapunov-like functions are special cases of generalized Lyapunov-like functions. According to this definition, a generalized Lyapunov-like function may grow both on $\left[t_{2 j}, t_{2 j+1}\right), \forall j \in N$, and on $E(T)$. In order to make a generalized Lyapunov-like function to be useful, the two types of growth must be measured and constrained properly. The growth of $V$ on $\left(t_{2 j}, t_{2 j+1}\right)$ is already bounded by a function $\phi \in C\left[R_{+}, R_{+}\right]$in the sense of $V(x(t)) \leq \phi\left(V\left(x\left(t_{2 j}\right)\right)\right), t \in$ $\left(t_{2 j}, t_{2 j+1}\right)$. To deal with the growth of $V$ on $E(T)$, we need the concept of class $\mathcal{G K}$ functions given below. 
Definition 3.7. A function $\alpha: R_{+} \rightarrow R_{+}$is called a class $\mathcal{G} \mathcal{K}$ function if it is increasing and right continuous at the origin with $\alpha(0)=0$.

It is easy to see from the definition that a class $\mathcal{K}$ function must be a class $\mathcal{G K}$ function. However, class $\mathcal{G K}$ functions are a much broader class than class $\mathcal{K}$.

With the help of class $\mathcal{G} \mathcal{K}$ functions, stability of switched systems via multiple Lyapunov functions can be characterized by the following proposition.

Proposition 3.8. Consider system (1) with $u_{\sigma} \equiv 0$. Suppose there exist continuous positive definite functions $V_{i}(x), i=$ $1,2, \ldots, m$, all defined around the origin and such that $V_{i}(0)=0$ and $V_{i_{k}}\left(x\left(t_{k}\right)\right) \geq V_{i_{k}}(x(t))$ for $t \in\left[t_{k}, t_{k+1}\right)$. Then, the origin of the system (1) is stable if and only if there exists a class $\mathcal{G} \mathcal{K}$ function $\alpha$ satisfying

$V_{i_{k}}\left(x\left(t_{k}\right)\right) \leq \alpha\left(\left\|x_{0}\right\|\right), k \geq 0$,

Proof. Trivial.

Though this proposition gives a necessary and sufficient condition for stability, it is almost useless because it can be used neither to test stability nor to guide the switching law design.

In the stability study of switched systems via multiple Lyapunov functions, a basic methodology is to test stability by using the information about change of each Lyapunov function at different corresponding activate intervals (Branicky, 1998; Hou et al., 1996; Ye et al., 1998). This allows us to test stability by comparing the values of each Lyapunov function at the times when the corresponding subsystem becomes active during the system evolution, separately for each subsystem index. More importantly, in some cases, this methodology provides a practical principle of designing switching laws. For example, the well-known "min-switching" strategy is based on this. Motivated by this consideration, we present a necessary and sufficient condition of stability by means of the change of each general Lyapunov-like function at different corresponding active intervals.

Theorem 3.9. Suppose that for each $i \in M$, there exists a positive definite generalized Lyapunov-like function $V_{i}(x)$ with respect to $f_{i}(x, 0)$ and the associated trajectory. Then,

(i) the origin of the system (1) with $u_{\sigma} \equiv 0$ is stable if and only if there exist class $\mathcal{G} \mathcal{K}$ functions $\alpha_{j}$ satisfying

$$
\begin{aligned}
V_{j}\left(x\left(t_{j_{k+1}}\right)\right)-V_{j}\left(x\left(t_{j_{1}}\right)\right) \leq & \alpha_{j}\left(\left\|x_{0}\right\|\right), \\
& k \geq 1, j=1,2, \ldots, m,
\end{aligned}
$$

(ii) the origin of the system (1) is asymptotically stable if and only if (5) holds and there exists $j$, such that $V_{j}\left(x\left(t_{j_{k}}\right)\right) \rightarrow 0$ as $k \rightarrow \infty$.

Proof. We first prove (i). Let $\phi_{i}$ be given by the generalized Lyapunov-like function $V_{i}(x)$ that is,

$V_{i_{k}}(x(t)) \leq \phi_{i_{k}}\left(V_{i_{k}}\left(x\left(t_{k}\right)\right)\right), \quad t_{k} \leq t<t_{k+1}$.

Sufficiency. For any constants $c, c_{1}, c_{2}>0, c_{1} \leq c_{2}$, let

$$
B(c)=\{x \mid\|x\| \leq c\}
$$

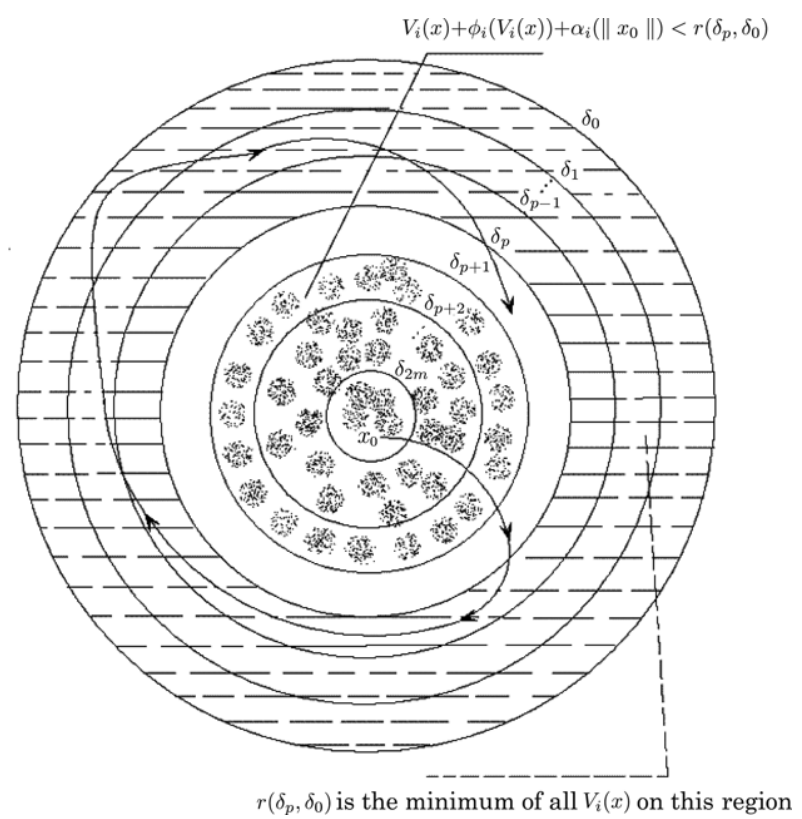

Fig. 1. The proof of Theorem 3.9.

and

$r\left(c_{1}, c_{2}\right)=\min \left\{V_{i}(x) \mid c_{1} \leq\|x\| \leq c_{2}, i \in M\right\}$.

Obviously, $r\left(c_{1}, c_{2}\right)$ is a positive continuous function of variables $c_{1}$ and $c_{2}$. Now, for any $\epsilon>0$, since $\alpha_{i}, V_{i}$ and $\phi_{i}$ are continuous with $\alpha_{i}(0)=0, V_{i}(0)=0$ and $\phi_{i}(0)=0$, it is always possible to select $\delta_{1}>0, \delta_{1}<\epsilon$, such that

$V_{i}(x)+\phi_{i}\left(V_{i}(x)\right)+\alpha_{i}\left(\left\|x_{0}\right\|\right)<r(\epsilon, \epsilon), \quad \forall x, x_{0} \in B\left(\delta_{1}\right)$.

For this $\delta_{1}>0$, we use the same procedure to choose $\delta_{2}>0$, $\delta_{2}<\delta_{1}$ such that

$$
\begin{aligned}
V_{i}(x)+\phi_{i}\left(V_{i}(x)\right)+\alpha_{i}\left(\left\|x_{0}\right\|\right)< & r\left(\delta_{1}, \epsilon\right) \\
& \text { when } x, x_{0} \in B\left(\delta_{2}\right) .
\end{aligned}
$$

Repeating the same procedure up to $2 m$ steps, we finally have $\epsilon=\delta_{0}>\delta_{1}>\delta_{2}>\cdots>\delta_{2 m}>0$ with the property as depicted in Fig. 1:

$V_{i}(x)+\phi_{i}\left(V_{i}(x)\right)+\alpha_{i}\left(\left\|x_{0}\right\|\right)<r\left(\delta_{p}, \delta_{0}\right)$,

if $x, x_{0} \in B\left(\delta_{p+1}\right), 0 \leq p \leq 2 m-1, \quad \forall i$.

We will show that $x(t) \in B(\epsilon)$ for any $t \in[0, \infty)$ if $x_{0} \in B\left(\delta_{2 m}\right)$ and thus stability follows.

For any $k \geq 0$, let $R_{k}$ be the number of the different elements of the set

$\left\{i_{0}, i_{1}, \ldots, i_{k}\right\} \bigcap\{1,2, \ldots, m\}$,

which is actually the total number of different subsystems that have ever been active for some time on $\left[t_{0}, t_{k+1}\right) .\left\{R_{k}, k=\right.$ $0,1, \ldots\}$ is obviously a nondecreasing sequence bounded by $m$. We can know by (6) that if $x_{k}=x\left(t_{k}\right) \in B\left(\delta_{q}\right)$ for some $k$ and $q$, then $x(t) \in B\left(\delta_{q-1}\right), t \in\left[t_{k}, t_{k+1}\right]$. In fact, the generalized Lyapunov-like function $V_{i_{k}}$ gives that

$V_{i_{k}}(x(t)) \leq \phi_{i_{k}}\left(V_{i_{k}}\left(x\left(t_{k}\right)\right)\right)<r\left(\delta_{q-1}, \delta_{0}\right)$.

Now, we claim that for any $k \geq 1$ :

(a) if $R_{k}=R_{k-1}+1$, then 
$V_{i_{k}}\left(x_{k}\right)+\phi_{i_{k}}\left(V_{i_{k}}\left(x_{k}\right)\right)+\alpha_{i_{k}}\left(\left\|x_{0}\right\|\right)<r\left(\delta_{2 m-2 R_{k}+2}, \delta_{0}\right)$,

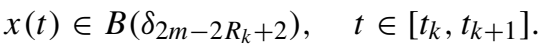

(b) if $R_{k}=R_{k-1}$, then

$$
\begin{aligned}
& V_{i_{k}}\left(x_{k}\right)+\phi_{i_{k}}\left(V_{i_{k}}\left(x_{k}\right)\right)+\alpha_{i_{k}}\left(\left\|x_{0}\right\|\right)<r\left(\delta_{2 m-2 R_{k}+1}, \delta_{0}\right), \\
& x(t) \in B\left(\delta_{2 m-2 R_{k}+1}\right), \quad t \in\left[t_{k}, t_{k+1}\right] .
\end{aligned}
$$

Note that $R_{0}=1$. According to the construction of $B\left(\delta_{2 m}\right)$, we have

$V_{i_{0}}\left(x_{0}\right)+\phi_{i_{0}}\left(V_{i_{0}}\left(x_{0}\right)\right)+\alpha_{i_{0}}\left(\left\|x_{0}\right\|\right)<r\left(\delta_{2 m-1}, \delta_{0}\right)$,

which gives that

$V_{i_{0}}(x(t)) \leq \phi_{i_{0}}\left(V_{i_{0}}\left(x_{0}\right)\right)<r\left(\delta_{2 m-1}, \delta_{0}\right)$.

Thus, $x(t) \in B\left(\delta_{2 m-1}\right), t \in\left[t_{0}, t_{1}\right]$. We prove the claim by induction on $k$. If $k=1$, then $R_{1}=2=R_{0}+1$. Since $x_{1}=x\left(t_{1}\right) \in B\left(\delta_{2 m-1}\right)$, (6) tells us that

$$
\begin{aligned}
V_{i_{1}}\left(x_{1}\right)+\phi_{i_{1}}\left(V_{i_{1}}\left(x_{1}\right)\right)+\alpha_{i_{1}}\left(\left\|x_{0}\right\|\right) & <r\left(\delta_{2 m-2}, \delta_{0}\right) \\
& =r\left(\delta_{2 m-2 R_{1}+2}, \delta_{0}\right) .
\end{aligned}
$$

Therefore,

$$
\begin{aligned}
V_{i_{1}}(x(t)) & \leq \phi_{i_{1}}\left(V_{i_{1}}\left(x_{1}\right)\right) \\
& <r\left(\delta_{2 m-2 R_{1}+2}, \delta_{0}\right), \quad t \in\left[t_{1}, t_{2}\right],
\end{aligned}
$$

which guarantees that $x(t) \in B\left(\delta_{2 m-2 R_{1}+2}\right), t \in\left[t_{1}, t_{2}\right]$. The claim holds.

Now, suppose the claim is true for $k \leq p$. Note that in any case of $R_{p}=R_{p-1}+1$ or $R_{p}=R_{p-1}$, we have $x(t) \in$ $B\left(\delta_{2 m-2 R_{p}+1}\right), t \in\left[t_{p}, t_{p+1}\right]$. If $R_{p+1}=R_{p}+1$, we have

$$
\begin{aligned}
& V_{i_{p+1}}\left(x_{p+1}\right)+\phi_{i_{p+1}}\left(V_{i_{p+1}}\left(x_{p+1}\right)\right)+\alpha_{i_{p+1}}\left(\left\|x_{0}\right\|\right) \\
& \quad<r\left(\delta_{2 m-2 R_{p}}, \delta_{0}\right)=r\left(\delta_{2 m-2 R_{p+1}+2}, \delta_{0}\right), \\
& x(t) \in B\left(\delta_{2 m-2 R_{p+1}+2}\right), \quad t \in\left[t_{p+1}, t_{p+2}\right] .
\end{aligned}
$$

The claim is true. If $R_{p+1}=R_{p}$, then, $p \geq 1, R_{p} \geq 2$ must hold because $R_{0}=1, R_{1}=2$. We split the proof into two subcases: $i_{p+1}=i_{0}$ and $i_{p+1} \neq i_{0}$.

If $i_{p+1}=i_{0}$, noticing $x_{0} \in B\left(\delta_{2 m}\right)$, (5) gives rise to

$V_{i_{p+1}}\left(\left(x_{p+1}\right)\right) \leq V_{i_{0}}\left(x_{0}\right)+\alpha_{i_{0}}\left(\left\|x_{0}\right\|\right)<r\left(\delta_{2 m-1}, \delta_{0}\right)$.

In view of $x_{p+1} \in B\left(\delta_{2 m-2 R_{p}+1}\right)$ and the definition of $r\left(\delta_{2 m-1}, \delta_{0}\right)$, we have $x_{p+1} \in B\left(\delta_{2 m-1}\right) \subseteq B\left(\delta_{2 m-2 R_{p+1}+2}\right)$. Thus,

$$
\begin{aligned}
& V_{i_{p+1}}\left(x_{p+1}\right)+\phi_{i_{p+1}}\left(V_{i_{p+1}}\left(x_{p+1}\right)\right)+\alpha_{i_{p+1}}\left(\left\|x_{0}\right\|\right) \\
& \quad<r\left(\delta_{2 m-2 R_{p+1}+1}, \delta_{0}\right), \\
& x(t) \in B\left(\delta_{2 m-2 R_{p+1}+1}\right), \quad t \in\left[t_{p+1}, t_{p+2}\right] .
\end{aligned}
$$

If $i_{p+1} \neq i_{0}$, then there must exist an integer $s \geq 1$ satisfying $p>s, i_{p+1}=i_{s}$ and $R_{s}=R_{s-1}+1$. That means that $t_{s}$ is the time when the $i_{p+1}$ th subsystem is activated for the first time. According to the assumption of the induction,

$V_{i_{s}}\left(x_{s}\right)+\phi_{i_{s}}\left(V_{i_{s}}\left(x_{s}\right)\right)+\alpha_{i_{s}}\left(\left\|x_{0}\right\|\right)<r\left(\delta_{2 m-2 R_{s}+2}, \delta_{0}\right)$.

Again, because of (5), it holds that

$V_{i_{p+1}}\left(x_{p+1}\right) \leq V_{i_{s}}\left(x_{s}\right)+\alpha_{i_{s}}\left(\left\|x_{0}\right\|\right)<r\left(\delta_{2 m-2 R_{s}+2}, \delta_{0}\right)$.
Therefore, $x_{p+1} \in B\left(\delta_{2 m-2 R_{s}+2}\right) \subseteq B\left(\delta_{2 m-2 R_{p+1}+2}\right)$, which in turn gives

$$
\begin{aligned}
& V_{i_{p+1}}\left(x_{p+1}\right)+\phi_{i_{p+1}}\left(V_{i_{p+1}}\left(x_{p+1}\right)\right)+\alpha_{i_{p+1}}\left(\left\|x_{0}\right\|\right) \\
& \quad<r\left(\delta_{2 m-2 R_{p+1}+1}, \delta_{0}\right) .
\end{aligned}
$$

In any cases the claim is true for $k=p+1$. Thus, $x(t) \in$ $B(\epsilon)$ for any $t \in[0, \infty)$.

Necessity. Suppose that the origin of the system (1) with $u_{\sigma} \equiv 0$ is stable. Let

$\alpha_{j}(s)=\sup _{k \geq 1,\left\|x_{0}\right\| \leq s}\left\{0, V_{j}\left(x\left(t_{j_{k+1}}\right)\right)-V_{j}\left(x\left(t_{j_{1}}\right)\right)\right\}$.

It follows from stability of the system that $\alpha_{j}(s)$ is locally welldefined and (5) holds. We only need show $\alpha_{j}(s)$ is a class $\mathcal{G K}$ function. $\alpha_{j}(s)$ is obviously increasing and satisfies $\alpha(0)=0$. While the right continuity at the origin follows from stability of the system.

Now, we show (ii). The proof of necessity is straightforward. Sufficiency follows from the fact that stability implies boundedness of both $x(t)$ and $\dot{x}(t)$ (left and right derivatives at $t_{k}$ ), which in turn guarantees uniform continuity of $x(t)$. Note that $V_{j}\left(x\left(t_{j_{k}}\right)\right) \rightarrow 0$ implies $x\left(t_{j_{k}}\right) \rightarrow 0$ as $k \rightarrow \infty$, which yields $x(t)$ tending to the origin due to uniform continuity and stability. This completes the proof.

Remark 3.10. If we knew $V_{j}\left(x\left(t_{j_{1}}\right)\right)$ could be set "small enough" by letting the initial state $x\left(t_{0}\right)$ be close to the origin, Theorem 3.9 would be trivial. However, we have no apriori knowledge that $V_{j}\left(x\left(t_{j_{1}}\right)\right)$ can be set "small enough" because the switching law can be arbitrary: time dependent, state dependent, or both, or even determined by a hybrid logic-based controller. Consequently, we have no idea about when and how the $j$ th subsystem is activated for the first time.

The meaning of Theorem 3.9 is that stability is ensured as long as $V_{j}\left(x\left(t_{j_{k+1}}\right)\right)-V_{j}\left(x\left(t_{j_{1}}\right)\right)$, which is the change of $V_{j}$ between any "switched on" time $t_{j_{k+1}}$ and the first active time $t_{j_{1}}$, is bounded by a class $\mathcal{G K}$ function, regardless of where $V_{j}\left(x\left(t_{j_{1}}\right)\right)$ is. This is not true for the general case of nongeneralized Lyapunov-like functions. In fact, one can easily construct an example where for some $j, V_{j}\left(x\left(t_{j_{1}}\right)\right)$ can be arbitrarily large though $x_{0}$ is set arbitrarily close to the origin. Thus, stability is lost.

Remark 3.11. (5) can be equivalently rewritten as

$\sum_{q=1}^{k}\left(V_{j}\left(x\left(t_{j_{q+1}}\right)\right)-V_{j}\left(x\left(t_{j_{q}}\right)\right)\right) \leq \alpha_{j}\left(\left\|x_{0}\right\|\right)$,

for any integer $k \geq 1, j=1,2, \ldots, m$.

Note that $V_{j}\left(x\left(t_{j_{q+1}}\right)\right)-V_{j}\left(x\left(t_{j_{q}}\right)\right)$ stands for the change of the same function $V_{j}(x)$ at two adjacent "switched on" times. Thus, (23) means that $V_{j}$ is allowed to grow on $E(\Sigma \mid j)$ but the total such change on any finite time interval should be bounded from above by a class $\mathcal{G K}$ function. As a special case, when Branicky's "nonincreasing" condition holds, that is, $V_{j}\left(x\left(t_{j_{q+1}}\right)\right)-V_{j}\left(x\left(t_{j_{q}}\right)\right) \leq 0,(23)$ is automatically satisfied with $\alpha_{j}=0$. 
Remark 3.12. In Theorem 3.9, no decreasing requirement of $V_{j}(x(t))$ on the corresponding active intervals is needed. Instead, we only need that $V_{j}(x(t))$ on an active interval does not exceed the value of some function of $V_{j}$ at the "switched on" time.

Remark 3.13. Other kinds of decreasing conditions like $\dot{V}_{i_{k}}(x(t))<0, t \in\left[t_{k}, t_{k+1}\right)$ or (3) definitely guarantee asymptotic stability. On the other hand, LaSalle type conditions might be preferable. In particular, the newly developed concepts of norm-observability (Hespanha et al., 2005) are helpful to achieve asymptotic stability. We will see this in the sequel.

To illustrate Theorem 3.9, we consider an example.

Example 3.14. Consider the switched linear system

$\dot{x}=A_{\sigma} x$

with three subsystems: $A_{1}=\left(\begin{array}{cc}0 & -2 \\ \frac{1}{2} & 0\end{array}\right), A_{2}=\left(\begin{array}{cc}0 & -3 \\ \frac{1}{3} & 0\end{array}\right)$, $A_{3}=\left(\begin{array}{cc}0 & -1 \\ 1 & 0\end{array}\right)$.

Obviously, none of the subsystems is asymptotically stable. Let $q_{j}(t)$ be the total number of times that the $j$ th subsystem is active before time $t$. The switching law is given by:

$\sigma(t)=\left\{\begin{array}{c}1, \quad \text { if } x(t) \text { is in the first, or second or } \\ \text { third quadrants, } \\ 2, \quad \text { if } q_{2}(t) \leq q_{3}(t)+1 \text { and } \\ x(t) \text { is in the fourth quadrant, } \\ 3, \quad \text { if } q_{2}(t)>q_{3}(t)+1 \text { and } \\ x(t) \text { is in the fourth quadrant, }\end{array}\right.$

Choose generalized Lyapunov-like functions as:

$V_{1}(x)=\frac{1}{2} x_{1}^{2}+2 x_{2}^{2}$,

$V_{2}(x)=\frac{1}{3} x_{1}^{2}+3 x_{2}^{2}, \quad V_{3}(x)=x_{1}^{2}+x_{2}^{2}$.

It is easy to see that $V_{i}(x(t))$ remains constant when the $i$ th subsystem is active and thus qualifies as a generalized Lyapunov-like function. Also, the Branicky's nonincreasing condition on the switched on time sequences is not satisfied. A straightforward calculation shows that (5) holds for $\alpha_{1}(s)=$ $9 s^{2}, \alpha_{2}(s)=6 s^{2}$ and $\alpha_{3}(s)=s^{2}$. Thus, the system (24) is stable by Theorem 3.9. For asymptotic stability, we have

$V_{1}\left(x\left(t_{1_{k+1}}\right)\right)-V_{1}\left(x\left(t_{1_{k}}\right)\right)=$

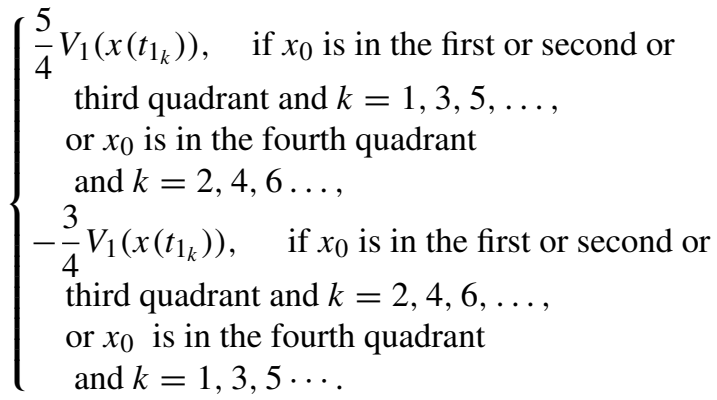

Thus $V_{1}\left(x\left(t_{1_{k+1}}\right)\right) \rightarrow 0$ as $k \rightarrow \infty$. Asymptotic stability follows. The system trajectory with the initial state $x_{0}=(2,0)^{T}$ is depicted in Fig. 2.

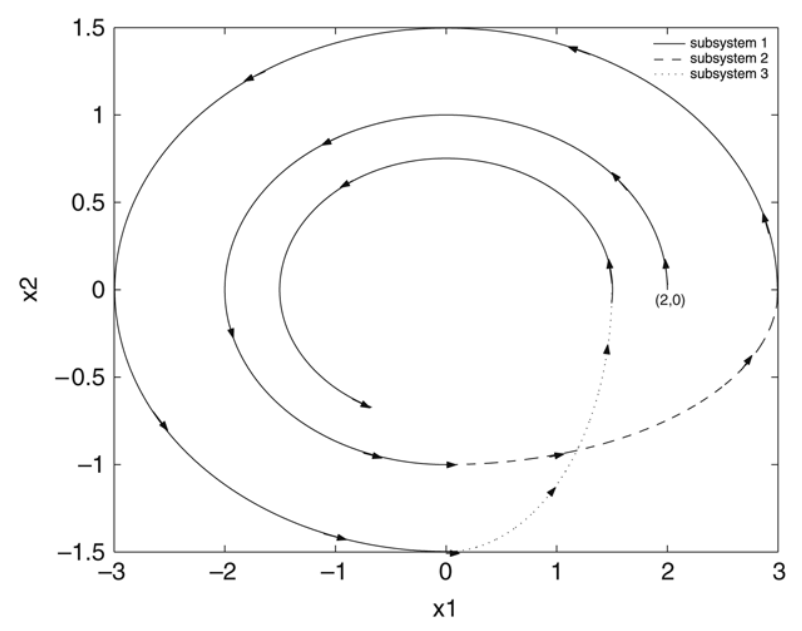

Fig. 2. Trajectory of the switched system (24).

\subsection{Alternative stability conditions}

Sometimes, instead of finding a class $\mathcal{G K}$ function, it might be convenient to check uniform convergence of certain series as shown in the following.

Theorem 3.15. Suppose for each $i \in M$, there exists a generalized Lyapunov-like function $V_{i}(x)$ with respect to $f_{i}(x, 0)$ and the associated trajectory. Then, the origin of the system (1) with $u_{\sigma} \equiv 0$ is stable if any of the following conditions, which are not equivalent, is satisfied:

(A) For any $j \in M$, the series

$A_{j}\left(x_{0}\right)=\sum_{p=1}^{\infty} \max \left\{0, V_{j}\left(x\left(t_{j_{p+1}}\right)\right)-V_{j}\left(x\left(t_{j_{p}}\right)\right)\right\}$

is convergent uniformly with respect to the initial state $x_{0}$ in a neighborhood of the origin;

(B) For any $j \in M$, the series

$B_{j}\left(x_{0}\right)=\sum_{p=1}^{\infty}\left(V_{j}\left(x\left(t_{j_{p+1}}\right)\right)-V_{j}\left(x\left(t_{j_{p}}\right)\right)\right)$

is convergent uniformly with respect to the initial state $x_{0}$ in a neighborhood of the origin;

(C) $V_{i_{k}}(x(t)) \leq V_{i_{k}}\left(x\left(t_{t_{k}}\right)\right), t \in\left[t_{k}, t_{k+1}\right)$, and there exists $a$ class $\mathcal{G} \mathcal{K}$ function $\alpha$ such that

$$
\begin{aligned}
C_{k}\left(x_{0}\right) & =\sum_{p=0}^{k}\left(V_{i_{p+1}}\left(x\left(t_{p+1}\right)\right)-V_{i_{p}}\left(x\left(t_{p+1}\right)\right)\right) \\
& \leq \alpha\left(\left\|x_{0}\right\|\right), \quad \forall k,
\end{aligned}
$$

or a little stronger condition, the series

$C\left(x_{0}\right)=\sum_{p=0}^{\infty}\left(V_{i_{p+1}}\left(x\left(t_{p+1}\right)\right)-V_{i_{p}}\left(x\left(t_{p+1}\right)\right)\right)$

is convergent uniformly with respect to the initial state $x_{0}$ in a neighborhood of the origin; 
(D) There exist class $\mathcal{G} \mathcal{K}$ functions $\alpha_{j}(\cdot)$ such that for any $k>1$

$$
\begin{aligned}
V_{j}\left(x\left(t_{j_{k+1}}\right)\right)-V_{j}\left(x\left(t_{j_{1}}\right)\right) & =\sum_{p=1}^{k}\left(V_{j}\left(x\left(t_{j_{p+1}}\right)\right)-V_{j}\left(x\left(t_{j_{p}}\right)\right)\right) \\
& \leq \alpha_{j}\left(\left\|x_{0}\right\|\right), \quad \forall j \in M .
\end{aligned}
$$

Proof. We show the following implications:

$$
(\mathrm{A}) \Rightarrow \quad \begin{aligned}
& \text { (B) } \Rightarrow \\
& (\mathrm{C}) \Rightarrow
\end{aligned} \quad \text { (D) } \Rightarrow \text { stability }
$$

$(\mathrm{A}) \Rightarrow(\mathrm{B})$. Since $A_{j}\left(x_{0}\right)$ is uniformly convergent, similarly to the proof of Theorem 3.9, we know that $V_{j}\left(x\left(t_{j_{1}}\right)\right)$ is uniformly bounded with respect to the initial state $x_{0}$ in a neighborhood of the origin. Since

$$
\begin{aligned}
& V_{j}\left(x\left(t_{j_{k+1}}\right)\right)-V_{j}\left(x\left(t_{j_{k}}\right)\right) \\
& \quad \leq \max \left\{0, V_{j}\left(x\left(t_{j_{k+1}}\right)\right)-V_{j}\left(x\left(t_{j_{k}}\right)\right)\right\},
\end{aligned}
$$

it is easy to know that $V_{j}\left(x\left(t_{j_{k+1}}\right)\right)$ is uniformly bounded with respect to the initial state $x_{0}$. Therefore, we can deduce from the uniform convergence of $A_{j}\left(x_{0}\right)$ that

$$
\begin{aligned}
V_{j} & \left(x\left(t_{j_{k+1}}\right)\right)-V_{j}\left(x\left(t_{j_{1}}\right)\right) \\
= & \sum_{p=1}^{k}\left(V_{j}\left(x\left(t_{j_{p+1}}\right)\right)-V_{j}\left(x\left(t_{j_{p}}\right)\right)\right)
\end{aligned}
$$

is uniformly convergent with respect to the initial state $x_{0}$ as $k \rightarrow \infty$.

(B) $\Rightarrow$ (D). According to the uniform convergence of $B_{j}\left(x_{0}\right)$, for any $\epsilon>0$, we can choose an integer $K_{j}>0$, which is independent of $x_{0}$, such that for any integers $p, q, K_{j} \leq p \leq$ $q \leq \infty$, and any initial state $x\left(t_{0}\right)$ in a neighborhood of the origin, it holds that

$$
\left|\sum_{k=p}^{q}\left(V_{j}\left(x\left(t_{j_{k+1}}\right)\right)-V_{j}\left(x\left(t_{j_{k}}\right)\right)\right)\right|<\epsilon .
$$

Let $R=\max _{1 \leq j \leq m}\left\{j_{K_{j}}\right\}$. Similarly to the proof of Theorem 3.9, we can choose a neighborhood $U_{\epsilon}$ of the origin such that $V_{i_{l}}\left(x\left(t_{l}\right)\right) \leq \epsilon$ if $x(0) \in U_{\epsilon}$ and $i_{l} \leq R$. This implies that the function $\alpha_{j}(s)=$ $\sup _{k \geq 1,\left\|x_{0}\right\| \leq s}\left\{0, V_{j}\left(x\left(t_{j_{k+1}}\right)\right)-V_{j}\left(x\left(t_{j_{1}}\right)\right)\right\}$ is well-defined around the origin and qualifies as a class $\mathcal{G K}$ function. It is trivial to check (29).

(C) $\Rightarrow$ (D). Let $\beta(r)=\max _{1 \leq i \leq m,\|x\| \leq r}\left\{V_{i}(x)\right\}$. It follows from (27) that for any $q \geq 1$,

$$
\begin{aligned}
V_{i_{q}}\left(x\left(t_{q}\right)\right)= & V_{i_{0}}\left(x\left(t_{0}\right)\right)+\sum_{p=1}^{q}\left(V_{i_{p}}\left(x\left(t_{p}\right)\right)-V_{i_{p-1}}\left(x\left(t_{p}\right)\right)\right) \\
& +\sum_{p=0}^{q-1}\left(V_{i_{p}}\left(x\left(t_{p+1}\right)\right)-V_{i_{p}}\left(x\left(t_{p}\right)\right)\right) \\
\leq & V_{i_{0}}\left(x\left(t_{0}\right)\right)+\sum_{p=1}^{q}\left(V_{i_{p}}\left(x\left(t_{p}\right)\right)-V_{i_{p-1}}\left(x\left(t_{p}\right)\right)\right) \\
\leq & \alpha\left(\left\|x_{0}\right\|\right)+\beta\left(\left\|x_{0}\right\|\right) .
\end{aligned}
$$

Therefore, (D) holds with $\alpha_{j}=2(\alpha+\beta)$ which is obviously a class $\mathcal{G K}$ function.

(D) $\Rightarrow$ stability. It follows from Theorem 3.9.

Remark 3.16. If $V_{i_{k}}$ and $V_{i_{k+1}}$ are connected at $t_{k+1}$, i.e. $V_{i_{k}}\left(x\left(t_{k+1}\right)\right)=V_{i_{k+1}}\left(x\left(t_{k+1}\right)\right)$, which is suggested by the well-known "min-switching" law $\sigma(t)=$ $\arg \min _{1 \leq i \leq m}\left\{V_{i}(x(t))\right\}$, the condition (C) is automatically satisfied. It is obvious that the condition (C) is much weaker than the adjacent $V_{i_{k}}$ and $V_{i_{k+1}}$ being connected at the switching times because we only need the boundedness of the sequence (27). This gives us considerable freedom in the switching law design, i.e. rather than following the "min-switching" law.

\subsection{Stabilizing switching law}

In this subsection, we address the issue of how to design a switching law to achieve stability with the help of the stability conditions given in Theorems 3.9 and 3.15.

First of all, we present a generalization of the well-known "min-switching" law (Liberzon \& Morse, 1999).

Suppose that we have continuous functions $V_{i}(x), \mu_{i j}(x)$, $i, j=1,2, \ldots, m$, satisfying

$\mu_{i j}(x)+\mu_{j k}(x) \leq \min \left\{0, \mu_{i k}(x)\right\}, \quad \forall i, j, k$.

Then, it is easy to know that for any integers $i_{1}, i_{2}, \ldots, i_{q} \in$ $\{1,2, \ldots, m\}$,

$\mu_{i_{1} i_{2}}(x)+\mu_{i_{2} i_{3}}(x)+\cdots+\mu_{i_{q-1} i_{q}}(x)+\mu_{i_{q} i_{1}}(x) \leq 0$.

Let

$\Omega_{i}=\left\{x \mid V_{i}(x)-V_{j}(x)+\mu_{i j}(x) \leq 0, j=1,2, \ldots, m\right\}$,

$\tilde{\Omega}_{i j}=\left\{x \mid V_{i}(x)-V_{j}(x)+\mu_{i j}(x)=0\right\}, \quad j \neq i$.

Then, the set $\tilde{\Omega}_{i}=\bigcup_{j=1, j \neq i}^{m} \tilde{\Omega}_{i j}$ contains the boundary of $\Omega_{i}$. We now show $\bigcup_{i=1}^{m} \Omega_{i}=R^{n}$. In fact, if this is false, namely, there exists $x \in R^{n}$ satisfying $x \notin \Omega_{i}, \forall i$, then we have an integer $q$ and a sequence $i_{1}, \ldots, i_{q}, i_{k} \neq i_{k+1}, k=1, \ldots, q$ with $i_{q+1}$ being considered as $i_{1}$, such that

$V_{i_{k}}(x)-V_{i_{k+1}}(x)+\mu_{i_{k} i_{k+1}}(x)>0$.

Taking the sum over $k$ and noticing (32) yields

$\sum_{k=1}^{q}\left(V_{i_{k}}(x)-V_{i_{k+1}}(x)+\mu_{i_{k} i_{k+1}}\right)=\sum_{k=1}^{q} \mu_{i_{k} i_{k+1}}(x) \leq 0$,

which contradicts (35).

The sets $\Omega_{i}$ have the property that if $x \in \Omega_{i} \bigcap \tilde{\Omega}_{i j}$ for some $i, j$ and $x \in R^{n}$, then $x \in \Omega_{j}$. In fact, $x \in \Omega_{i} \cap \tilde{\Omega}_{i j}$ means that $V_{i}(x)-V_{k}(x)+\mu_{i k}(x) \leq 0$ for any $k$ and $V_{i}(x)-V_{j}(x)+$ $\mu_{i j}(x)=0$. Thus, $V_{j}(x)=V_{i}(x)+\mu_{i j}(x)$. This in turn gives

$$
\begin{aligned}
V_{j}(x)-V_{k}(x)+\mu_{j k}(x) & =V_{i}(x)-V_{k}(x)+\mu_{i j}(x)+\mu_{j k}(x) \\
& \leq V_{i}(x)-V_{k}(x)+\mu_{i k}(x) \leq 0,
\end{aligned}
$$

Now, we design the switching law as follows.

$\sigma(t)=i \quad$ if $\sigma\left(t^{-}\right)=i$ and $\quad x(t) \in$ int $\Omega_{i}$,

$\sigma(t)=j \quad$ if $\sigma\left(t^{-}\right)=i \quad$ and $\quad x(t) \in \tilde{\Omega}_{i j}$. 


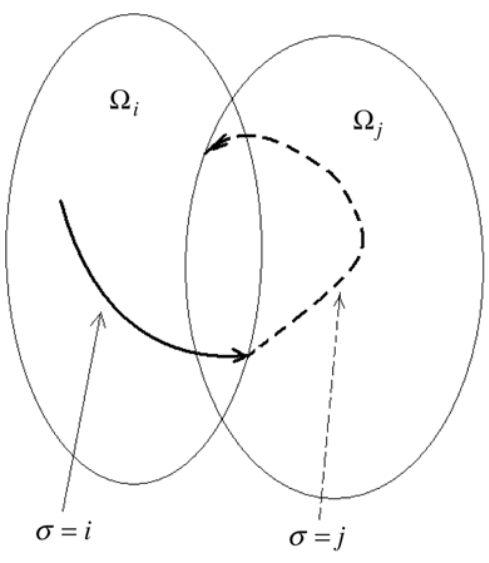

Fig. 3. The switching law (36).

Thus, once the trajectory enters $\Omega_{i}$ it will remain in $\Omega_{i}$ until it hits the boundary in $\tilde{\Omega}_{i j}$ and then enters $\Omega_{j}$. In other words, switching only takes place on $\tilde{\Omega}_{i j}$ for some $j$. The switching law is shown in Fig. 3.

Remark 3.17. Any set $\Omega_{i}$ with empty interior is excluded from the construction of the switching law (36). The switching from the $i$ th subsystem to the $j$ th subsystem only occurs on $\tilde{\Omega}_{i j}$. The switching law (36) is uniquely defined up to a set of measure zero. This problem can be easily fixed, for example, by the method in Liberzon (2003).

Theorem 3.18. Suppose that we have positive definite smooth functions $V_{i}(x)$ with $V_{i}(0)=0$, continuous functions $\beta_{i j}(x) \leq$ 0 , smooth functions $\mu_{i j}(x), i, j=1,2, \ldots, m$ with $\mu_{i j}(0)=0$ and $\mu_{i i}(x)=0$, such that

$$
\begin{aligned}
& \frac{\partial V_{i}}{\partial x} f_{i}(x, 0)+\sum_{j=1}^{m} \beta_{i j}(x)\left(V_{i}(x)-V_{j}(x)+\mu_{i j}(x)\right) \leq 0, \\
& \quad i=1,2, \ldots, m, \\
& \frac{\partial \mu_{i j}}{\partial x} f_{i}(x, 0) \leq 0, \quad i, j=1,2, \ldots, m \\
& \mu_{i j}(x)+\mu_{j k}(x) \leq \min \left\{0, \mu_{i k}(x)\right\}, \quad \forall i, j, k .
\end{aligned}
$$

Also, assume that the switching law (36) is applied and no sliding modes occur on $\tilde{\Omega}_{i j}$. Then, the origin of the system (1) with $u_{\sigma} \equiv 0$ is stable. Moreover, if the inequalities in (37) hold strictly for $x \neq 0$, asymptotic stability is assured.

Proof. Since $\beta_{i j}(x) \leq 0,(37)$ implies $\frac{\partial V_{i}}{\partial x} f_{i}(x, 0) \leq 0, x \in$ $\Omega_{i}, i=1,2, \ldots, m$, which together with (38) tell us that $V_{i_{k}}(x(t))$ and $\mu_{i_{k} j}(x(t))$ are all decreasing on $\left[t_{k}, t_{k+1}\right)$.

For $k \geq 0$, according to the switching law (36), at each switching time we have

$V_{i_{k+1}}\left(x\left(t_{k+1}\right)\right)-V_{i_{k}}\left(x\left(t_{k+1}\right)\right)=\mu_{i_{k} i_{k+1}}\left(x\left(t_{k+1}\right)\right)$.

Thus,

$$
\begin{aligned}
& \left(V_{i_{k+1}}\left(x\left(t_{k+1}\right)\right)-V_{i_{k}}\left(x\left(t_{k+1}\right)\right)\right) \\
& \quad+\left(V_{i_{k+2}}\left(x\left(t_{k+2}\right)\right)-V_{i_{k+1}}\left(x\left(t_{k+2}\right)\right)\right) \\
& =\mu_{i_{k} i_{k+1}}\left(x\left(t_{k+1}\right)\right)+\mu_{i_{k+1} i_{k+2}}\left(x\left(t_{k+2}\right)\right) \\
& \leq \mu_{i_{k} i_{k+1}}\left(x\left(t_{k+1}\right)\right)+\mu_{i_{k+1} i_{k+2}}\left(x\left(t_{k+1}\right)\right) \leq 0 .
\end{aligned}
$$

Therefore,

$$
\begin{aligned}
& \sum_{p=0}^{k}\left(V_{i_{p+1}}\left(x\left(t_{p+1}\right)\right)-V_{i_{p}}\left(x\left(t_{p+1}\right)\right)\right) \\
& \quad \leq \begin{cases}0, & \text { if } k \text { is odd } \\
\mu_{i_{0} i_{1}}\left(x\left(t_{1}\right)\right) \leq \mu_{i_{0} i_{1}}\left(x_{0}\right), & \text { if } k \text { is even. }\end{cases}
\end{aligned}
$$

Choose $\alpha(s)=\max _{\|x\| \leq s}\left\{\left|\mu_{i j}(x)\right|, 1 \leq i, j \leq m\right\}$, the result follows immediately from (C) in Theorem 3.15. Moreover, if the inequalities in (37) hold strictly for $x \neq 0$, asymptotic stability follows from the standard argument of Lyapunov theory.

Remark 3.19. When $\mu_{i j} \equiv 0, \forall i, j$, (38) and (39) are automatically satisfied and (37) becomes the well-known result in Liberzon (2003) and the switching law given by (36) degenerates exactly into the "min-switching" strategy. When $\mu_{i j} \not \equiv 0$ and the inequalities (38) are strict, $\mu_{i j}$ will tend to zero as $t$ goes to infinity. Therefore, roughly speaking, in a long run, the switching law will coincide with the "min-switching" law.

Remark 3.20. Theorem 3.18 is based on the assumption that the system state can be observed. When the state is not available, state observers or filters (Zhang, Shi, Wang, \& Gao, 2006) can be used.

Remark 3.21. For the switched linear system

$\dot{x}=A_{\sigma} x+B_{\sigma} u_{\sigma}$,

we may look for positive definite quadratic functions $V_{i}(x)=$ $x^{T} P_{i} x$, quadratic functions $\mu_{i j}(x)=x^{T} Q_{i j} x$ and constants $\beta_{i j}$. Thus (37)-(39) become, respectively, the following matrix inequalities

$$
\begin{aligned}
& P_{i} A_{i}+A_{i}^{T} P_{i}+\sum_{j=1}^{m} \beta_{i j}\left(P_{i}-P_{j}+Q_{i j}\right) \leq 0, \\
& \quad i=1,2, \ldots, m, \\
& Q_{i j} A_{i}+A_{i}^{T} Q_{i j} \leq 0, \quad i, j=1,2, \ldots, m, \\
& Q_{i j}+Q_{j k}-Q_{i k} \leq 0, \quad \forall i, j, k, \\
& Q_{i j}+Q_{j k} \leq 0, \quad \forall i, j, k .
\end{aligned}
$$

\section{L -gain}

In this section, we will firstly give an $L_{2}$-gain description for switched systems and then investigate the issues of internal stability produced by $L_{2}$-gain and achieving $L_{2}$-gain by design of switching laws.

\subsection{Definitions}

Definition 4.1. The system (1) has weak $L_{2}$-gain $\gamma$ under the switching law $\Sigma$ if there exist positive definite continuous functions $V_{1}(x), V_{2}(x), \ldots, V_{m}(x)$ with $V_{i}(0)=0$, and class $\mathcal{G K}$ functions $\alpha_{j}$ such that 
(i)

$$
\begin{aligned}
& V_{i_{k}}(x(t))-V_{i_{k}}(x(s)) \\
& \quad \leq \int_{s}^{t}\left(\gamma^{2}\left\|u_{i_{k}}(\tau)\right\|^{2}-\left\|h_{i_{k}}(x(\tau))\right\|^{2}\right) \mathrm{d} \tau, \\
& t_{k} \leq s \leq t<t_{k+1}
\end{aligned}
$$

holds for any $u_{i_{k}}$ satisfying $\int_{t_{0}}^{T}\left\|u_{i_{k}}(t)\right\|^{2} \mathrm{~d} t<\infty, \forall T>0$, $k=1,2, \ldots$

(ii) When $u_{i}=0$, and for $j=1,2, \ldots, m$,

$$
\begin{aligned}
Q_{j}\left(x_{0}\right) & =\sum_{k=1}^{p}\left(V_{j}\left(x\left(t_{j_{k+1}}\right)\right)-V_{j}\left(x\left(t_{j_{k}+1}\right)\right)\right) \\
& \leq \alpha_{j}\left(\left\|x_{0}\right\|\right), \quad \forall p .
\end{aligned}
$$

Definition 4.2. The system (1) has strong $L_{2}$-gain $\gamma$ under the switching law $\Sigma$ if it has weak $L_{2}$-gain $\gamma$ and

$\int_{0}^{T}\left(\gamma^{2}\left\|u_{\sigma(t)}(t)\right\|^{2}-\left\|h_{\sigma(t)}(x(t))\right\|^{2}\right) \mathrm{d} t \geq 0$

holds for any $T>0$ when $x(0)=0$.

In the case that the switched system (1) has only one subsystem, the weak $L_{2}$-gain and strong $L_{2}$-gain properties merge into the usual $L_{2}$-gain property.

Remark 4.3. In Definition 4.1, (48) is the usual but localized $L_{2}$-gain property for the active subsystem on the corresponding time intervals. Again, Condition (ii) measures the change of functions $V_{j}$ when inactive.

Remark 4.4. If the "sequence nonincreasing condition" (Liberzon, 2003; Peleties \& DeCarlo, 1991) $V_{j}\left(x\left(t_{j_{k+1}}\right)\right)-$ $V_{j}\left(x\left(t_{j_{k}+1}\right)\right) \leq 0, j=1,2, \ldots, m$, holds, Condition (ii) in Definition 4.1 is automatically satisfied.

Remark 4.5. It is easy to see that the system (1) has strong $L_{2}$ gain $\gamma$ if Condition (i) and (ii) in Definition 4.1 hold and when $x(0)=0$ the function

$V(t)=\left\{\begin{array}{l}V_{i_{0}}(x(t)), \quad t \in\left[t_{0}, t_{1}\right) \\ V_{i_{k}}(x(t))-\sum_{j=1}^{k}\left(V_{i_{j}}\left(x\left(t_{j}\right)\right)-V_{i_{j-1}}\left(x\left(t_{j}\right)\right)\right), \\ \quad t \in\left[t_{k}, t_{k+1}\right), k=1,2, \ldots\end{array}\right.$

is nonnegative.

\subsection{Achieving $L_{2}$-gain via design of switching laws}

This subsection addresses the problem of how to achieve $L_{2}$ gain for the smooth affine switched system

$\dot{x}=f_{\sigma}(x)+g_{\sigma}(x) u_{\sigma}$,

$y=h_{\sigma}(x)$,

by design of switching laws. The key idea here is to adopt the same strategy developed in Theorem 3.18 in dealing with stability.
Theorem 4.6. Consider the switched system (52). Suppose that we have positive definite smooth functions $V_{i}(x)$ with $V_{i}(0)=$ 0 , continuous functions $\beta_{i j}(x) \leq 0$, smooth functions $\mu_{i j}(x)$ with $\mu_{i j}(0)=0$ and $\mu_{i i}(x)=0$, such that

$$
\begin{aligned}
& \frac{\partial V_{i}}{\partial x} f_{i}+\frac{1}{2 \gamma^{2}} \frac{\partial V_{i}}{\partial x} g_{i} g_{i}^{T} \frac{\partial^{T} V_{i}}{\partial x} \\
& \quad+\frac{1}{2} h_{i}^{T} h_{i}+\sum_{j=1}^{m} \beta_{i j}(x)\left(V_{i}(x)-V_{j}(x)+\mu_{i j}(x)\right) \leq 0 \\
& \quad i=1,2, \ldots, m \\
& \frac{\partial \mu_{i j}}{\partial x} f_{i}(x) \leq 0, \quad i, j=1,2, \ldots, m, \\
& \mu_{i j}(x)+\mu_{j k}(x) \leq \min \left\{0, \mu_{i k}(x)\right\}, \quad \forall i, j, k
\end{aligned}
$$

Then, under the switching law (36), the system (52) has weak $L_{2}$-gain. If in addition,

$\frac{\partial \mu_{i j}}{\partial x} g_{i}=0, \quad \forall i, j$

then, system (52) has strong $L_{2}$-gain.

Proof. Under the switching law (36), it is easy to show

$$
\begin{aligned}
\frac{\partial V_{i_{k}}}{\partial x} & f_{i_{k}}+\frac{1}{2 \gamma^{2}} \frac{\partial V_{i_{k}}}{\partial x} g_{i_{k}} g_{i_{k}}^{T} \frac{\partial^{T} V_{i_{k}}}{\partial x}+\frac{1}{2} h_{i_{k}}^{T} h_{i_{k}} \leq 0, \\
t_{k} & \leq t<t_{k+1} .
\end{aligned}
$$

Similarly to the proof of Theorem 3.18 , we can prove that (48) holds and when $u_{i}=0$ (49) also holds. Therefore, weak $L_{2}$ gain follows. We now show strong $L_{2}$-gain. In view of the switching law, (54) and (56), when the $i$ th subsystem is active, it holds that

$$
\begin{aligned}
\dot{\mu}_{i j}(x(t)) & =\frac{\partial \mu_{i j}}{\partial x}\left(f_{i}(x(t))+g_{i}(x(t)) u_{i}\right) \\
& =\frac{\partial \mu_{i j}}{\partial x} f_{i}(x(t)) \leq 0, \quad i=1,2, \ldots, m .
\end{aligned}
$$

Then all reasoning in the proof of Theorem 3.18 still holds. In particular, when $x_{0}=0$, (42) gives

$$
\sum_{p=0}^{k}\left(V_{i_{p+1}}\left(x\left(t_{p+1}\right)\right)-V_{i_{p}}\left(x\left(t_{p+1}\right)\right)\right) \leq \mu_{i_{0} i_{1}}\left(x_{0}\right)=0, \quad \forall k,
$$

which means the function defined by (51) is nonnegative, and thus strong $L_{2}$-gain follows.

Remark 4.7. For the switched linear system

$$
\begin{aligned}
& \dot{x}=A_{\sigma(t)} x+B_{\sigma(t)} u_{\sigma(t)} \\
& y=C_{\sigma(t)} x
\end{aligned}
$$

and quadratic Lyapunov functions $V_{i}(x)=\frac{1}{2} x^{T} P_{i} x$ with positive definite matrices $P_{i}$, we need to solve the following matrix inequalities

$$
\begin{aligned}
& P_{i} A_{i}+A_{i}^{T} P_{i}+\frac{1}{\gamma^{2}} P_{i} B_{i} B_{i}^{T} P_{i}+C_{i}^{T} C_{i} \\
& \quad+\sum_{j=1}^{m} \beta_{i j}\left(P_{i}-P_{j}+Q_{i j}\right) \leq 0, \quad i, j=1,2, \ldots, m,
\end{aligned}
$$


$Q_{i j} A_{i}+A_{i}^{T} Q_{i j} \leq 0, \quad i, j=1,2, \ldots, m$,

$Q_{i j}+Q_{j k}-Q_{i k} \leq 0, \quad \forall i, j, k$,

$Q_{i j}+Q_{j k} \leq 0, \quad \forall i, j, k$,

where $Q_{i j}$ are symmetric matrices. If we want strong $L_{2}$-gain, $Q_{i j}$ need to satisfy $Q_{i j} B_{i}=0, \forall i, j$.

\subsection{Stability analysis induced by $L_{2}$-gain}

As $L_{2}$-gain of nonswitched systems gives internal stability, $L_{2}$-gain of switched systems is also expected to imply similar stability properties. This will be shown in the following.

Proposition 4.8. If the system (1) has weak $L_{2}$-gain $\gamma$ under the switching law $\Sigma$, then the origin of the system (1) with $u_{i}=0$ is stable.

Proof. Condition (i) in Definition 4.1 with $u_{i}=0$ means that $V_{i_{k}}(x(t))$ is decreasing on $\left[t_{k}, t_{k+1}\right)$ and thus qualifies as a generalized Lyapunov-like function. Note that $V_{j}\left(x\left(t_{j_{k+1}}\right)\right)-$ $V_{j}\left(x\left(t_{j_{k}+1}\right)\right) \geq V_{j}\left(x\left(t_{j_{k+1}}\right)\right)-V_{j}\left(x\left(t_{j_{k}}\right)\right)$. Condition (ii) guarantees that the sequence $\left\{V_{j}\left(x\left(t_{j_{k}}\right)\right), k=1,2, \ldots,\right\}$ is bounded uniformly with respect to the initial state $x_{0}$. It is not difficult to show that (5) in Theorem 3.9 is satisfied. Thus, stability follows.

To achieve asymptotic stability, many types of conditions can be imposed. Here, we consider some conditions of the LaSalle invariance type. To this end, we need the newly developed concept of small-time norm-observability.

Definition 4.9 (Hespanha et al., 2005). The nonlinear system

$\dot{x}=f(x)$,

$y=h(x)$

is called small-time norm-observable if for any $\tau>0$ there exists a class $\mathcal{K}_{\infty}$ function $\gamma$ such that $\|x(t)\| \leq \gamma\left(|y|_{[t, t+\tau]}\right)$ holds for any $x\left(t_{0}\right)$ and $t \geq t_{0}$, where $|y|_{[t, t+\tau]}=\operatorname{ess} \sup \{\|$ $y(s) \| \mid s \in[t, t+\tau]\}$.

Theorem 4.10. Suppose the system (1) has weak $L_{2}$-gain $\gamma$ under the switching law $\Sigma$. If $V_{i}(x), i=1,2 \ldots, m$ are globally defined positive definite radially unbounded functions, and there exists $j$ with $\lim _{k \rightarrow \infty}\left(t_{j_{k}+1}-t_{j_{k}}\right) \neq 0$ and the corresponding subsystem with zero input is small-time normobservable, then, the origin of the system (1) with $u_{i}=0$ is globally asymptotically stable.

Proof. Stability follows from Proposition 4.8. Now, we show the global attractiveness. The following proof is motivated by Hespanha et al. (2005).

For the $j$ satisfying $\lim _{k \rightarrow \infty}\left(t_{j_{k}+1}-t_{j_{k}}\right) \neq 0$, there exists $\delta>0$ such that the set $\Lambda=\left\{k \mid t_{j_{k}+1}-t_{j_{k}} \geq \delta\right\}$ is infinite. Consider the auxiliary function

$\tilde{y}_{j}(t)=\left\{\begin{array}{l}y_{j}(x(t)), \quad t \in \bigcup_{k \in \Lambda}\left[t_{j_{k}}, t_{j_{k}+1}\right), \\ 0, \quad \text { otherwise. }\end{array}\right.$
For any $t>0$, if $t_{j_{k}} \leq t<t_{j_{k}+1}$ holds for some $k \in \Lambda$, it follows from (48) and (49) that

$$
\begin{aligned}
& \int_{t_{0}}^{t} \tilde{y}_{j}^{T}(\tau) \tilde{y}_{j}(\tau) \mathrm{d} \tau \leq \sum_{p=1}^{k-1}\left(V_{j}\left(x\left(t_{j_{p}}\right)\right)-V_{j}\left(x\left(t_{j_{p}+1}\right)\right)\right) \\
& \quad+V_{j}\left(x\left(t_{j_{k}}\right)\right)-V_{j}(x(t)) \\
& \leq V_{j}\left(x\left(t_{j_{1}}\right)\right)+\sum_{p=1}^{k-1}\left(V_{j}\left(x\left(t_{j_{p+1}}\right)\right)-V_{j}\left(x\left(t_{j_{p}+1}\right)\right)\right) \\
& \leq V_{j}\left(x\left(t_{j_{1}}\right)\right)+\alpha_{j}\left(\left\|x_{0}\right\|\right)<\infty,
\end{aligned}
$$

where $\alpha_{j}$ is the $\mathcal{G} \mathcal{K}$ function satisfying (49).

For any $t>0$, if $t \notin \bigcup_{k \in \Lambda}\left[t_{j_{k}}, t_{j_{k}+1}\right)$, there exists $k \in \Lambda$ such that $t \geq t_{j_{k}+1}$ and $t<t_{j_{q}}$ for any $q \in \Lambda$ and $q>k$. In this case, we have $\tilde{y}_{j}(s) \equiv 0, s \in\left[t_{j_{k}+1}, t\right]$, and (66) still holds. Note that Condition (i) and (ii) in Definition 4.1 guarantee the boundedness of $x(t)$, which in turn implies that the derivative (left and right derivatives at switching times $t_{k}$ ) is also bounded. Hence, $\tilde{y}_{j}(t)$ is uniformly continuous over $\bigcup_{k \in \Lambda}\left[t_{j_{k}}, t_{j_{k}+1}\right)$. In view of $t_{j_{k}+1}-t_{j_{k}} \geq \delta, k \in \Lambda$ and (66), we have $\tilde{y}_{j}(t) \rightarrow 0$ as $t \rightarrow \infty$. It follows from small-time norm-observability of the $j$ th subsystem that $x\left(t_{j_{k}}\right) \rightarrow 0$ as $k \rightarrow \infty$ and $k \in \Lambda$. Thus, $x(t) \rightarrow 0$ as $t \rightarrow \infty$ due to stability of the closed-loop system and continuity of $x(t)$.

\section{5. $H_{\infty}$ control}

This section concentrates on the $H_{\infty}$ control problem via state feedback and switching law design. For simplicity, we consider a switched system, which is affine both in control input $u_{\sigma}$ and disturbance input $\omega_{\sigma}$, described by

$\dot{x}=f_{\sigma}(x)+g_{\sigma}(x) u_{\sigma}+p_{\sigma}(x) \omega_{\sigma}$,

$y=h_{\sigma}(x)$.

The $H_{\infty}$ control problem for the switched system (67) is stated as follows:

Problem 5.1. Let a constant $\gamma>0$ be given. Find, if possible, a continuous state feedback controller $u_{i}=u_{i}(x)$ for each subsystem, and a switching law $\sigma(t)$ (if the switching law is to be designed), such that:

(a) The origin of the closed-loop system is globally asymptotically stable when $\omega_{i} \equiv 0$.

(b) The overall $L_{2}$-gain in usual sense from $\omega_{\sigma(t)}$ to $\tilde{y}=$ $\tilde{h}_{\sigma(t)}(x)=\left(h_{\sigma(t)}(x), u_{\sigma(t)}(x)\right)^{T}$ on any finite time interval $[0, T]$ is less than or equal to $\gamma$, i.e. if $x(0)=0$, it holds that

$J_{T}=\int_{0}^{T}\left(\left\|\tilde{h}_{\sigma(t)}(x(t))\right\|^{2}-\gamma^{2}\left\|\omega_{\sigma(t)}(t)\right\|^{2}\right) \mathrm{d} t \leq 0$.

Remark 5.2. Unlike the $L_{2}$-gain description in Section 4 , where we adopt weak and strong forms to characterize gain features of individual subsystems when active and total gain properties over any finite time interval, respectively, the $H_{\infty}$ control problem is formulated here in a unified form to maintain consistency with nonswitched systems. 
The $H_{\infty}$ control problem is obviously solvable if there exists a positive definite function $V(x)$ such that the inequality

$$
\begin{aligned}
& V(x(t))-V\left(x\left(t_{0}\right)\right) \\
& \quad \leq \frac{1}{2} \int_{t_{0}}^{t}\left(\gamma^{2}\left\|\omega_{\sigma(\tau)}(\tau)\right\|^{2}-\left\|\tilde{h}_{\sigma(\tau)}(x(\tau))\right\|^{2}\right) \mathrm{d} \tau
\end{aligned}
$$

holds. This property is standard for general nonlinear systems, but is much too restrictive for switched systems in two ways. Firstly, for a switched system, each subsystem usually has its individual attenuation level $\gamma_{i}$ when this subsystem is active. Secondly, each individual subsystem has its own function $V_{i}(x)$. A common function $V(x)$ for all subsystems is difficult to find or might not exist at all. The first one can be easily fixed by taking the maximum over all $\left\{\gamma_{i}\right\}$ to give a common level; while conservative, this is always possible. For the second one, it is reasonable and necessary to adopt multiple $V$ functions instead of seeking a single one for all subsystems. However, this leads to the following two problems: (a) internal stability property may be lost; (b) the total attenuation level may be weakened by an exponential decay rate $\lambda$ (Zhai et al., 2001):

$\int_{0}^{T} \mathrm{e}^{-\lambda t}\|\tilde{y}(x(t))\|^{2} \mathrm{~d} t \leq \gamma^{2} \int_{0}^{T}\left\|\omega_{\sigma(t)}(t)\right\|^{2} \mathrm{~d} t$.

Our solutions will avoid these problems. It is clear that achieving strong $L_{2}$-gain plus global asymptotic stability gives rise to a solution to the $H_{\infty}$ control problem. The idea here is to achieve strong $L_{2}$-gain via state feedback and switching law design.

\subsection{Solvability under fixed switching law}

Theorem 5.3. Suppose there exist positive definite smooth proper functions (see van der Schaft (2000) for the definition) $V_{i}(x), i=1, \ldots, m$, satisfying the partial differential inequalities

$$
\begin{aligned}
\tilde{f}_{i_{k}}(x)= & \frac{\partial V_{i_{k}}}{\partial x} f_{i_{k}}+\frac{1}{2} \frac{\partial V_{i_{k}}}{\partial x}\left(\frac{1}{\gamma^{2}} p_{i_{k}} p_{i_{k}}^{T}-g_{i_{k}} g_{i_{k}}^{T}\right) \\
& \times \frac{\partial^{T} V_{i_{k}}}{\partial x}+\frac{1}{2} h_{i_{k}}^{T} h_{i_{k}} \leq 0, \quad t_{k} \leq t<t_{k+1} .
\end{aligned}
$$

For the ith subsystem, design the continuous state feedback controller

$u_{i}=u_{i}(x)=-g_{i}^{T}(x) \frac{\partial^{T} V_{i}}{\partial x}(x), \quad 1 \leq i \leq m$.

If (1) for $x\left(t_{0}\right)=0$ and any integer $N$, it holds that

$$
\sum_{k=0}^{N}\left(V_{i_{k+1}}\left(x\left(t_{k+1}\right)\right)-V_{i_{k}}\left(x\left(t_{k+1}\right)\right)\right) \leq 0,
$$

(2) for $\omega_{\sigma(t)} \equiv 0$ and some class $\mathcal{G K}$ functions $\alpha_{j}$, it holds that

$$
\sum_{k=1}^{p}\left(V_{j}\left(x\left(t_{j_{k+1}}\right)\right)-V_{j}\left(x\left(t_{j_{k}+1}\right)\right)\right) \leq \alpha_{j}\left(\left\|x_{0}\right\|\right),
$$

$\forall p \geq 1, j \in M$,
(3) there exists a closed-loop subsystem with $\omega_{\sigma(t)} \equiv 0$, which has infinitely many active intervals of length at least $\delta$ between switching times and is small-time norm-observable,

then the $H_{\infty}$ control problem is solved by the feedback (71).

Proof. When $T \in\left[t_{k}, t_{k+1}\right)$, using a method similar to that in van der Schaft (2000), we have

$$
\begin{aligned}
J_{T} \leq & 2 \sum_{j=0}^{k-1}\left(V_{i_{j+1}}\left(x\left(t_{j+1}\right)\right)-V_{i_{j}}\left(x\left(t_{j+1}\right)\right)\right) \\
& +2\left(V_{i_{0}}\left(x\left(t_{0}\right)\right)-V_{i_{k}}(x(T))\right) .
\end{aligned}
$$

When $x\left(t_{0}\right)=0$, applying (72) gives rise to $J_{T} \leq$ $-2 V_{i_{k}}(x(T)) \leq 0$, which means that the closed-loop system has strong $L_{2}$-gain $\gamma$. Similarly to the proof of Theorem 4.10, we can easily derive global asymptotic stability when $\omega_{j} \equiv 0$ by small-time norm-observability.

Remark 5.4. If the switched system has only one subsystem, Theorem 5.3 degenerates into the well-known result for nonswitched systems in terms of the Hamilton-Jacobi inequality (Helton \& James, 1999; van der Schaft, 2000).

\subsection{Simultaneous design of controllers and switching law}

Next, we consider how to solve the $H_{\infty}$ control problem by simultaneous design of state feedback and switching laws.

Theorem 5.5. Consider the switched system (67). Suppose that we have positive definite and proper smooth functions $V_{i}(x)$ with $V_{i}(0)=0$, continuous functions $\beta_{i}(x) \leq 0$, smooth functions $\mu_{i j}(x)$ with $\mu_{i j}(0)=0$ and $\mu_{i i}(x)=0$, such that

$$
\begin{aligned}
& \frac{\partial V_{i}}{\partial x} f_{i}+\frac{1}{2} \frac{\partial V_{i}}{\partial x}\left(\frac{1}{\gamma^{2}} p_{i} p_{i}^{T}-g_{i} g_{i}^{T}\right) \frac{\partial^{T} V_{i}}{\partial x}+\frac{1}{2} h_{i}^{T} h_{i} \\
& +\sum_{j=1}^{m} \beta_{i j}(x)\left(V_{i}(x)-V_{j}(x)+\mu_{i j}(x)\right) \leq 0, \quad \forall i, \\
& \frac{\partial \mu_{i j}}{\partial x}\left(f_{i}(x)-g_{i}(x) g_{i}^{T}(x) \frac{\partial^{T} V_{i}}{\partial x}(x)\right) \leq 0, \\
& i, j=1,2, \ldots, m, \\
& \mu_{i j}(x)+\mu_{j k}(x) \leq \min \left\{0, \mu_{i k}(x)\right\}, \quad \forall i, j, k, \\
& \frac{\partial \mu_{i j}}{\partial x} p_{i}=0, \quad \forall i, j .
\end{aligned}
$$

Design the feedback controllers $u_{i}(x)=-g_{i}^{T}(x) \frac{\partial^{T} V_{i}}{\partial x}(x)$ and the switching law given by (36). If Condition (3) in Theorem 5.3 holds, then the $H_{\infty}$ control problem is solved.

Proof. Applying Theorem 4.6 to the closed-loop system immediately gives the results.

\section{Concluding remarks}

We have given a necessary and sufficient condition for stability of switched systems in terms of multiple generalized Lyapunov-like functions. This condition tells us how much the corresponding general Lyapunov-like function is allowed to 
grow on the "switched on" time sequence without violating stability. Using this condition we do not need to worry when and how each subsystem is activated for the first time.

The $L_{2}$-gain description and analysis proposed are based on the consideration of change of value of associated $V$ functions when being inactive. This change represents a kind of energy exchange from an active subsystem to an inactive one. The boundedness requirement of such energy exchange is reasonable in order to maintain stability.

A solution to the $H_{\infty}$ control problem for switched systems has been presented. A sufficient condition for the problem to be solvable has been derived by using multiple Lyapunov functions that may not be connected at switching times. Each Lyapunov function satisfies a partial differential inequality, which is a localized form of the Hamilton-Jacobi inequality for the usual nonlinear $H_{\infty}$ control problem for each active time interval. The continuous state feedback controllers for all subsystems are designed once a set of solutions to the partial differential inequalities are available.

Compared with the Hamilton-Jacobi inequality, these partial differential inequalities are easier to solve because they are only needed to be satisfied on corresponding active time intervals. This can be seen more clearly in the case that the switching is state partition based. Only "semi-global" (according to the corresponding active region) solutions to these differential inequalities are needed. Although finding such "semi-global" solutions is much easier than finding global solutions, there is no systematic way to solve the partial differential inequalities proposed in this paper. Effective approaches need to be investigated for the general nonlinear case.

The results provide more freedom for the problems addressed to be solvable. In fact, in the case of a given switching law, we have more Lyapunov function candidates for each subsystem because they are no longer necessarily connected one to another at switching points and are allowed to grow on "switched on" time sequences. In the case of designing switching laws, for any fixed or chosen set of Lyapunov function candidates for all subsystems, if the switching law given by the "min-switching" strategy cannot solve the problem, we can solve algebraic inequalities as shown in Theorems 3.18, 4.6 and 5.5, to obtain some Lyapunov functions that are disconnected at switching points. Furthermore, arbitrarily adjusting the switching strategy as long as the total "jumps" satisfy the conditions can provide possible solutions. It is worth pointing out that in this case, in order to meet the boundedness of the total "jumps", a hybrid switching strategy might be more suitable, that is, when and how the switching takes place may depend on factors such as state, time and the previous "jumps" record.

\section{Acknowledgements}

This work was supported by the Australian Research Council Federation Fellowship and Centres of Excellence Schemes and the National Natural Science Foundation of China under Grant 60574013. Part of the work was supported by the
Research Grants Council of Hong Kong when the authors were at the City University of Hong Kong.

\section{References}

Bacciotti, A. (2004). Stabilization by means of state space depending switching rules. Systems \& Control Letters, 53(3), 195-201.

Banerjee, A., Arkun, Y., Pearson, R., \& Ogunnaike, B. (1997). $H_{\infty}$ control of nonlinear processes using multiple linear models. In R. Murray-Smith, \& T. A. Johansen (Eds.), Multiple model approaches to modeling and control (pp. 293-305). London: Taylor \& Francis.

Branicky, M. S. (1998). Multiple Lyapunov functions and other analysis tools for switched and hybrid systems. IEEE Transactions on Automatic Control, 43(4), 475-482.

Ceragioli, F. (2006). Finite valued feedback laws and piecewise classical solutions. Nonlinear Analysis: Theory, Methods \& Applications, 65(5), 984-998.

Cheng, D., Guo, L., Lin, Y., \& Wang, Y. (2005). Stabilization of switched linear systems. IEEE Transactions on Automatic Control, 50(5), 661-666.

Cronin, B., \& Spong, M. W. (2003). Switching control for multi-input cascade nonlinear systems. In Proceedings of the 42nd IEEE conference on decision and control (pp. 4277-4282).

Cuzzola, F. A., \& Morari, M. (2002). An LMI approach for $H_{\infty}$ analysis and control of discrete-time piecewise affine systems. International Journal of Control, 75(16), 1293-1301.

Daafouz, J., Riedinger, P., \& Iung, C. (2002). Stability analysis and control synthesis for switched systems: A switched Lyapunov function approach. IEEE Transactions on Automatic Control, 47(11), 1883-1887.

Decarlo, R. A., Branicky, M. S., Pettersson, S., \& Lennartson, B. (2000). Perspectives and results on the stability and Stabilizability of hybrid systems. In Proceedings of the IEEE: Special issue on hybrid systems (pp. 1069-1082). New York: IEEE Press.

EI-Farra, N. H., Mhaskar, P., \& Christofides, P. D. (2005). Output feedback control of switched nonlinear systems using multiple Lyapunov functions. Systems \& Control Letters, 54(12), 1163-1182.

Haddad, W. M., \& Chellaboina, V. (2001). Dissipativity theory and stability of feedback interconnections for hybrid dynamical systems. Mathematical Problems in Engineering, 7(4), 299-335.

Helton, J. W., \& James, M. R. (1999). Extending $H_{\infty}$ control to nonlinear systems. Philadelphia, PA: SIAM.

Hespanha, J. P. (1998). Logic-based switching algorithms in control. Ph.D. thesis, Dept. of Electrical Engineering, Yale University.

Hespanha, J. P., Liberzon, D., Angeli, D., \& Sontag, E. D. (2005). Nonlinear norm observability notions and stability of switched systems. IEEE Transactions on Automatic Control, 50(2), 154-168.

Hespanha, J. P., \& Morse, A. S. (1999). $L_{2}$-induced gains of switched linear systems. In V. D. Blondel, E. D. Sontag, M. Vidyasagar, \& J. C. Willems (Eds.), Open problems in mathematical systems theory and control (pp. 45-47). London: Springer-Verlag.

Hill, D. J., \& Moylan, P. J. (1976). The stabilityy of nonlinear dissipative systems. IEEE Transaction on Automatic Control, 21(5), 708-711.

Hill, D. J., \& Moylan, P. J. (1977). Stability results for nonlinear feedback systems. Automatica, 13(4), 377-382.

Hou, L., Michel, A. N., \& Ye, H. (1996). Stability analysis of switched systems. In Proceedings of the 35th IEEE conference on decision and control (pp. 120-1212).

Ishii, H., \& Francis, B. A. (2002). Stabilizing a linear system by switching control with dwell time. IEEE Transactions on Automatic Control, 47(12), 1962-1973.

Ji, Z., Wang, L., \& Guo, X. (2007). Design of switching sequences for controllability realization of switched linear systems. Automatica, 43(4), 662-668.

Kosmatopoulos, E. B., \& Ioannou, P. A. (2002). Robust switching adaptive control of multi-input nonlinear systems. IEEE Transactions on Automatic Control, 47(4), 610-624.

Liberzon, D. (2003). Switching in systems and control. Boston, MA: Birkhauser. 
Liberzon, D., \& Morse, A. S. (1999). Basic problems in stability and design of switched systems. IEEE Control Systems Magazine, 19(5), 59-70.

Lin, H., \& Antsaklis, P. J. (2003). Disturbance attenuation properties for discrete-time uncertain linear switched systems. In Proceedings of the 42nd IEEE conference on decision and control (pp. 5289-5294).

Margaliot, M. (2006). Stability analysis of switched systems using variational principles: An introduction. Automatica, 42(12), 2059-2077.

Michaletzky, G., \& Gerencser, L. (2002). BIBO stability of linear switching systems. IEEE Transactions Automatic Control, 47(11), 1895-1898.

Narendra, K. S., Driollet, O. A., Feiler, M., \& George, K. (2003). Adaptive control using multiple models, switching and tuning. International Journal of Adaptive Control \& Signal Processing, 17(2), 87-102.

Ooba, T., \& Funahashi, Y. (1997). On a common quadratic Lyapunov function for widely distant systems. IEEE Transactions on Automatic Control, 42(12), 1697-1699.

Peleties, P., \& DeCarlo, R. (1991). Asymptotic stability of m-switched systems using Lyapunov-like functions. In Proceedings of American control conference (pp. 1679-1684).

Savkin, A. V., Skafidas, E., \& Evans, R. J. (1999). Robust output feedback stabilizability via controller switching. Automatica, 35(1), 69-74.

Skafidas, E., Evans, R. J., Savkin, A. V., \& Petersen, I. R. (1999). Stability results for switched controller systems. Automatica, 35(4), 553-564.

Sun, Z., \& Ge, S. S. (2005). Switched linear systems - control and design. New York: Springer-Verlag.

Sun, X., Zhao, J., \& Hill, D. J. (2006). Stability and $L_{2}$-gain analysis for switched delay systems: Adelay-dependent method. Automatica, 42(10), 1769-1774.

van der Schaft, A. J. (2000). $L_{2}$-gain and passivity techniques in nonlinear control. New York: Springer.

Xie, D., Wang, L., Hao, F., \& Xie, G. (2004). LMI approach to $L_{2}$-gain analysis and control synthesis of uncertain switched systems. IEE Proceedings on Control Theory \& Applications, 151(1), 21-28.

Ye, H., Michel, A. N., \& Hou, L. (1998). Stability theory for hybrid dynamical systems. IEEE Transactions on Automatic Control, 43(4), 461-474.

Zhai, G., Ho, B., Yasuda, K., \& Michel, A. N. (2001). Disturbance attenuation properties of time-controlled switched systems. Journal of Franklin Institute, 338(7), 765-779.

Zhang, L., Shi, P., Wang, C., \& Gao, H. (2006). Robust $H_{\infty}$ filtering for switched linear discrete-time systems with polytopic uncertainties. International Journal of Adaptive Control and Signal Processing, 20(6), 291-304.

Zhang, L., Shi, P., Boukas, E.-K., \& Wang, C. (2006). $H_{\infty}$ control of switched linear discrete-time systems with polytopic uncertainties. Optimal Control, Applications and Methods, 27(5), 273-291.
Zhao, J., \& Dimirovski, G. M. (2004). Quadratic stability of a class of switched nonlinear systems. IEEE Transactions on Automatic Control, 49(4), 574-578.

Zhao, J., \& Hill, D. J. (2005). On stability and $L_{2}$-gain for switched systems. In Proceedings of the 44th IEEE conference on decision and control (pp. 3279-3284).

Zhao, J., \& Spong, M. W. (2001). Hybrid control for global stabilization of the cart-pendulum system. Automatica, 37(12), 1941-1951.

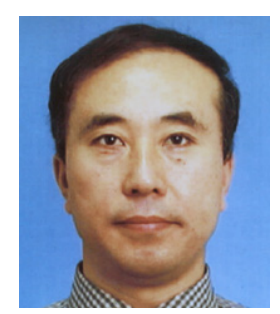

Jun Zhao was born in 1957. He received the B.S. and M.S. degrees in mathematics in 1982 and 1984 respectively, from Liaoning University, China. He completed his Ph.D. in Control Theory and Applications in 1991 at Northeastern University, China. From 1992 to 1993 he was a postdoctoral fellow at the same university. Since 1994 he has been with the School of Information Science and Engineering, Northeastern University, China, where he is currently a professor. From February 1998 to February 1999, he was a senior visiting scholar at the Coordinated Science Laboratory, University of Illinois at Urbana-Champaign. From November 2003 to May 2005, he was a Research Fellow at Department of Electronic Engineering, City University of Hong Kong. Since November 2006, as a Fellow, he has been with the Department of Information Engineering, Research School of Information Sciences and Engineering, The Australian National University.

His main research interests include switched systems, hybrid control, nonlinear systems and robust control.

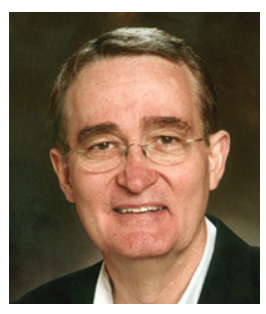

David J. Hill received the BE and BSc degrees from the University of Queensland, Australia, in 1972 and 1974, respectively. He received the $\mathrm{PhD}$ degree in Electrical Engineering from the University of Newcastle, Australia, in 1976. He is currently a Professor and Australian Research Council Federation Fellow in the Research School of Information Sciences and Engineering at The Australian National University. He has held academic and substantial visiting positions at the universities of Melbourne, California (Berkeley), Newcastle (Australia), Lund (Sweden), Sydney and Hong Kong (City University). He holds honorary professorships at the University of Sydney, University of Queensland (Australia), South China University of Technology, City University of Hong Kong, Wuhan University and Northeastern University (China). His research interests are in network systems science, stability analysis, nonlinear control and applications. He is a Fellow of the Institution of Engineers, Australia, the Institute of Electrical and Electronics Engineers, USA and the Australian Academy of Science; he is also a Foreign Member of the Royal Swedish Academy of Engineering Sciences. 